\title{
Estudio del factor de disipación dieléctrica como herramienta diagnóstica.
}

\section{Artículo Original}

Wilfredo Fariñas-Coronado ${ }^{1}$, Zenaida Paz ${ }^{1}$, Gregorio J. Orta ${ }^{2}$, Ernesto Rodrìguez-Denis ${ }^{3}$.

${ }^{1}$ Centro de Ingeniería Biomédica, Unexpo Puerto Ordaz, Venezuela, Puerto Ordaz, Edo. Bolivar, Venezuela. ${ }^{2}$ Hospital Uyapar, Puerto Ordaz, Servicio de Anatomía Patológica. ${ }^{3}$ Centro de Bioingeniería, ISPJAE, La Habana, Cuba.

\section{RESUMEN.}

Introducción. El estudio de las propiedades eléctricas de los tejidos biológicos data del siglo XIX. El desarrollo de esta línea de investigación y sus aplicaciones clínicas se han visto incrementadas en los últimos tiempos debido a la comprobada aplicación del análisis de las propiedades dieléctricas de los tejidos biológicos. En este artículo se presentan los valores del factor de disipación dieléctrica, en función de la frecuencia, de un conjunto de muestras de tejido mamario en condiciones sanas y patológicas.

Material y métodos. Las propiedades eléctricas de las muestras que se consideraron, capacitancia y conductancia, fueron medidas en un intervalo de frecuencia entre $10 \mathrm{~Hz}$ y $10 \mathrm{MHz}$ utilizando un analizador de impedancia HP4192A en modo logarítmico y calibrado para compensar las perdidas parásitas producto del arreglo de electrodos. La señal utilizada fue de $500 \mathrm{mV}$ rms, considerando un modelo RC en paralelo, un barrido logarítmico de frecuencia en 6 décadas y un modo de barrido de datos denominado AVERAGE disponible en el equipo analizador de impedancia. La adquisición se realizó en forma automática utilizando un software diseñado por el CIB-UNEXPO con plataforma LabView 5.01, denominado Z-Mammario Soft®.

Resultados y conclusiones. De los resultados se observa una marcada diferencia del factor de disipación dieléctrica entre los tejidos sanos y patológicos en el intervalo de frecuencia entre $1 \mathrm{KHz}$ y 1 MHz. (Rev Biomed 2002; 13:249-255)

Palabras clave: Factor de disipación dieléctrica, tejido mamario, bioimpedancia, propiedades eléctricas.

\section{SUMMARY.}

A study of the dielectric dissapation factor as a diagnostic tool.

Introduction. The study of the electrical properties of biological tissues dates from the nineteenth century. The development of this line of investigation and its clinical applications has increased recently due to the proved application of analysis of dielectric properties of biological tissues in this article, values of the dielectric dissipation factor are shown in terms of the frequency of a collection of samples of mammary tissue in both healthy and pathological conditions.

Solicitud de sobretiros: MsC. Wilfredo Farinas-Coronado, Centro de Ingeniería Biomédica, Unexpo Puerto Ordaz, Venezuela, apartado postal 1209, Puerto Ordaz, Edo. Bolivar,Venezuela. E-mail:wfarinas@poz.unexpo.edu, wfarinas@cantv.net. 


\section{W Fariñas-Coronado, Z Paz, GJ Orta, E Rodrìguez-Denis.}

Material and methods. The electric properties of the samples which were considered, capacitance and conductance, were measured at an interval of frequency between $10 \mathrm{~Hz}$ and $10 \mathrm{MHz}$ using a HP4192A impedance analyser in logarithmic mode and calibrated to compense for the parasitic loss, a product of the arrangement of electrodes. The signal used was $500 \mathrm{mVrms}$, considering a parallel RC model, a 6 decade frequency logarithmic sweep and a sweep made of data denominated AVERAGE available in the impedance analyser equipment. Acquisition was achieved automatically using software designed by CIB-UNEXPO with a Lab View 5.01 platform, called Z-Mammario Soft ${ }^{\circledR}$.

Results and conclusions. A considerable difference in the dielectric dissipation factor was observed between healthy and pathologic tissue in the interval of frequency between $1 \mathrm{KHz}$ and $1 \mathrm{MHz}$.

(Rev Biomed 2002; 13:249-255)

Key words: Dielectric dissipation factor, mammary tissue, bioimpedance, electric properties .

\section{INTRODUCCIÓN.}

La humanidad ha mostrado interés en los fenómenos eléctricos presentados por los seres vivos. Las primeras contribuciones en esta área, que hicieron posible mostrar que el tejido era un conductor y que su resistencia variaba con la frecuencia, datan de fines del siglo XIX. Estudios posteriores proporcionaron las bases para el estudio de la impedancia del tejido (bioimpedancia), demostraron su dependencia con la frecuencia y destacaron el movimiento de energía almacenada y disipación al nivel de la membrana del tejido (1-4). Formalmente, los estudios de las propiedades eléctricas de materiales biológicos comenzaron a desarrollarse en la década de 1920, cuando los biofísicos aplicaron la teoría de mezcla de Maxwell-Frick a estudios sobre dieléctricos heterogéneos. A partir de entonces, varios autores (5-8) han presentado resultados sobre estudios de propiedades eléctricas de tejidos biológicos demostrando que la membrana celular limita el paso de la corriente de baja frecuencia a través de la célula, pero permite el paso de la corriente de alta frecuencia; esto hace posible manifestar el aspecto capacitivo de la membrana implicado en los mecanismos de relajación al nivel de la interfaz membrana-electrolito (9). Herman P. Schwan y otros investigadores (1-7) estudiaron las propiedades eléctricas de diferentes tejidos biológicos y desarrollaron diversas técnicas para realizar las mediciones de la bioimpedancia, o impedancia biológica, a diferentes rangos de frecuencia (1-9).

El objetivo principal de caracterizar eléctricamente un tejido biológico radica en el hecho de conocer su comportamiento desde el punto de vista eléctrico. Estas propiedades y sus componentes tienen especial interés en:

a) Estudiar el efecto de las ondas electromagnéticas de alta frecuencia usadas en radar y técnicas de comunicación sobre los tejidos biológicos.

b) Determinar el comportamiento y las características de las moléculas que constituyen el tejido. Estas características son momento dipolar, tipo de molécula, etc.

c) Diagnóstico médico: Conociendo las propiedades eléctricas de los tejidos sanos y comparándolas con las características de tejidos anómalos, puede posibilitar que la bioimpedancia sea una herramienta diagnóstica auxiliar en algunos trastornos Biológicos, entre ellos el cáncer de mamas.

Esta aplicación ha sido la razón fundamental del desarrollo reciente de estudios de las propiedades eléctricas de tejidos biológicos y de su aplicación práctica en medicina (10-13).

Es bien conocido que los tejidos normales y anómalos difieren morfológicamente y puede presumirse que estas diferencias inducen cambios en las propiedades eléctricas de dichos tejidos $(8,13,16)$.

La mayoría de los cambios que se presentan en tejidos biológicos estudiados por medio de bioimpedancia eléctrica se hacen más evidentes en el intervalo de frecuencias comprendido entre $10 \mathrm{kHz}$ y $10 \mathrm{kHz}$ (17). Los cambios histológicos y citológicos en tejido canceroso, provocan modificaciones en el contenido de agua celular y de electrolitos en las 
Factor de disipación dieléctrica.

propiedades de la membrana celular, lo cual a su vez provoca cambios en las propiedades eléctricas de dicho tejido, permitiendo la posibilidad de identificar lesiones cancerosas $(13,17)$. Esta información se puede usar para calcular el factor de disipación dieléctrica para poder distinguir entre un tejido sano y uno canceroso.

\section{MATERIAL Y MÉTODOS.}

Para realizar este estudio se obtuvieron 16 muestras, cuyo espesor se muestra en el cuadro 1, de tejido mamario en acto quirúrgico de dieciséis intervenciones divididas de la siguiente manera: 8 cadáveres femeninos presumiblemente sin lesión. 4 intervenciones de masectomía 4 intervenciones de biopsias exploratorias.

A todas las muestras se le realizaron estudios histológicos para conocer sus condiciones histopatológicas, verificándose así lo antes afirmado. Las edades de las donantes estaban comprendidas entre 23 y 44 años. Estas muestras fueron limpiadas y colocadas en envases individuales con solución salina $\left(0.9 \% \mathrm{NaCl}\right.$ en $\mathrm{H}_{2} \mathrm{O}$ y $\left.\mathrm{pH}=6.10\right)$ y almacenadas a una temperatura de aproximadamente $4-5^{\circ} \mathrm{C}$ hasta el momento de la medición de los parámetros eléctricos. Las mediciones estos parámetros, capacitancia y conductancia, se realizaron a temperatura ambiente en un lapso comprendido entre 24 y 48 horas después de realizada la intervención quirúrgica. Para realizar la medición, una vez alcanzada la temperatura ambiente, las muestras se extraían de la solución salina, se removía el exceso de líquido y se colocaban en un arreglo de electrodos de $\mathrm{Ag} / \mathrm{AgCl}$ como el que se muestra en la figura 1 . Este procedimiento, similar a otro reportado por otros investigadores $(16,20,21)$, se mantuvo aproximadamente constante y siempre fue menor de $1 \mathrm{~min}$. La distancia entre los electrodos se obtuvo directamente de un equipo trazador de altura Digimatic Mitutoyo, modelo 192-630, Japón, el cual presenta la separación con una precisión de \pm $0.02 \mathrm{~mm}$. El cuadro 1 muestra los resultados del espesor de cada muestra.

Las propiedades eléctricas, capacitancia y
Cuadro 1

Espesor de las muestras en estudio.

\begin{tabular}{cc}
\hline $\mathrm{N}^{\circ}$ de la muestra & $\begin{array}{c}\text { Espesor del tejido mamario } \\
(\mathrm{mm}) \pm 0.02 \mathrm{~mm}\end{array}$ \\
\hline 1 & 2.06 \\
2 & 3.06 \\
3 & 0.66 \\
4 & 1.98 \\
5 & 1.83 \\
6 & 1.57 \\
7 & 1.12 \\
8 & 0.16 \\
9 & 1.70 \\
10 & 2.23 \\
11 & 0.09 \\
12 & 2.87 \\
13 & 1.81 \\
14 & 0.77 \\
15 & 0.93 \\
16 & 0.46 \\
\hline
\end{tabular}

conductancia, de estas muestras fueron medidas en un intervalo de frecuencia entre $10 \mathrm{~Hz}$ y $10 \mathrm{MHz}$ utilizando un analizador de impedancia HP4192A calibrado para compensar las perdidas parásitas. La señal utilizada fue de $500 \mathrm{mV}$ y considerando un modelo RSC en paralelo $(13,16,17,20,21)$. La adquisición se realizó en forma automática utilizando un software diseñado por el CIB-UNEXPO con plataforma LabView 5.01, denominado Z-Mamario Soft®. Con los datos obtenidos de la medición se realizaron los cálculos del factor de disipación

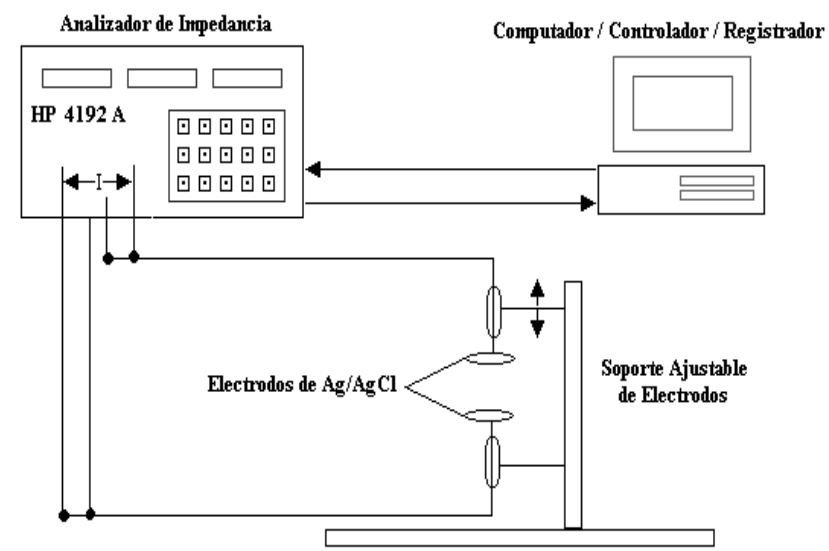

Figura 1.- Diagrama en bloque del sistema de medición utilizado.

Vol.13/No.4/Octubre-Diciembre, 2002 
W Fariñas-Coronado, Z Paz, GJ Orta, E Rodriguez-Denis.

dieléctrica de acuerdo a la siguiente relación (22):

$$
\tan \delta(\omega)=\frac{\varepsilon^{\prime \prime}(\omega)}{\varepsilon^{\prime}(\omega)},
$$

Donde: $e^{\prime}(w)$ representa la permitividad relativa, $e$ " (w) representa Factor de disipación dieléctrica relativa y d(w) el ángulo de disipacións dieléctricas.

El cálculo y el análisis de los resultados se realizó con el programa Sigmaplot 4.08. La fig. 1 muestra el sistema de medición utilizado.

\section{RESULTADOS.}

Para el cálculo del factor de disipación dieléctrica se se utilizó la ecuación 1 , considerando los valores medidos de conductancia, capacitancia y las características físicas de las muestras. La variación del factor de disipación dieléctrica de las muestras patológicas y sanas en función de la frecuencia se muestran en las Fig. 2 y Fig. 3, respectivamente. Asimismo, las Fig. 4 y Fig. 5 muestran las medias de ambos casos. En todos los casos se observa diferencia, significativa, sobre $100 \%$, entre el factor de perdida dieléctrica de los tejidos sanos y cancerosos. Las barras de dispersión indican los valores máximos y mínimos alrededor de los cuales se mantienen los valores de las diferentes muestras.

\section{DISCUSIÓN.}

Los resultados obtenidos reflejan claramente

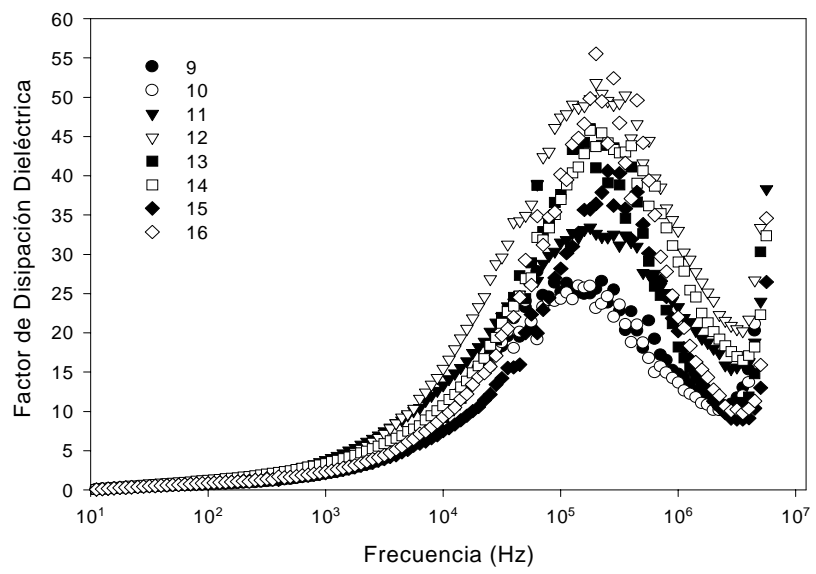

Figura 2.- Factor de Pérdida Dieléctrica de las muestras patológicas.

Revista Biomédica

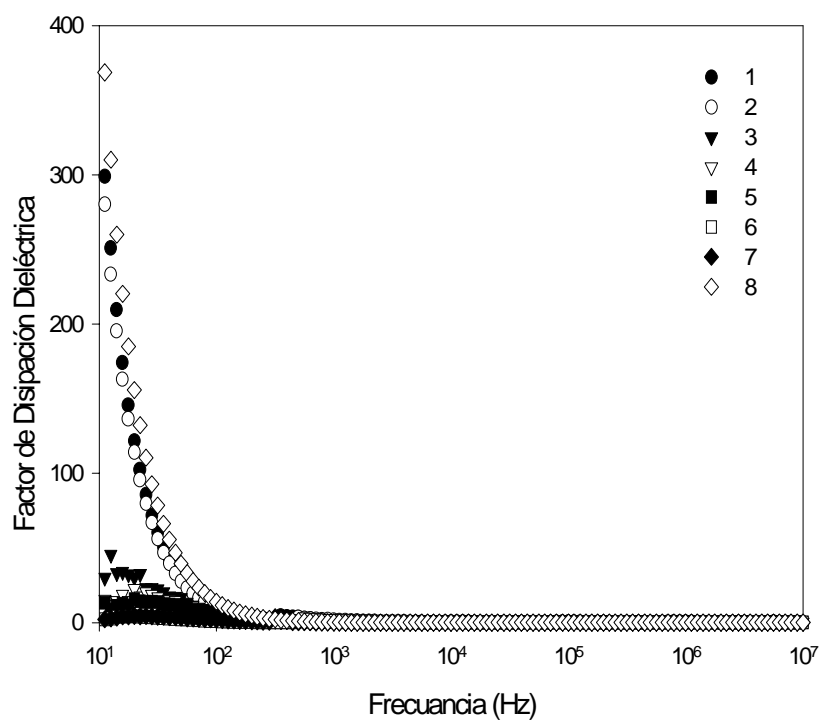

Figura 3.- Factor de Pérdida Dieléctrica de las muestras sanas.

que el factor de disipación dieléctrica de las muestras sanas es diferenciable del factor de disipación dieléctrica de las muestras patológicas, tal como se indica en la figuras anteriores. Esta diferencia, mayor al $100 \%$, es notable desde el punto de vista cualitativo y cuantitativo, tal como se muestra en los cuadros 2 y 3 , lo que pudiera indicar la posibilidad de diferenciar el tipo de patología presente en el tejido, lo cual es significativamente observable en las barras de dispersión mostradas en las figuras 4 y 5 . Nótese que estas barras no reflejan un error estadístico, pues no es este el caso, ya que no se consideran en este

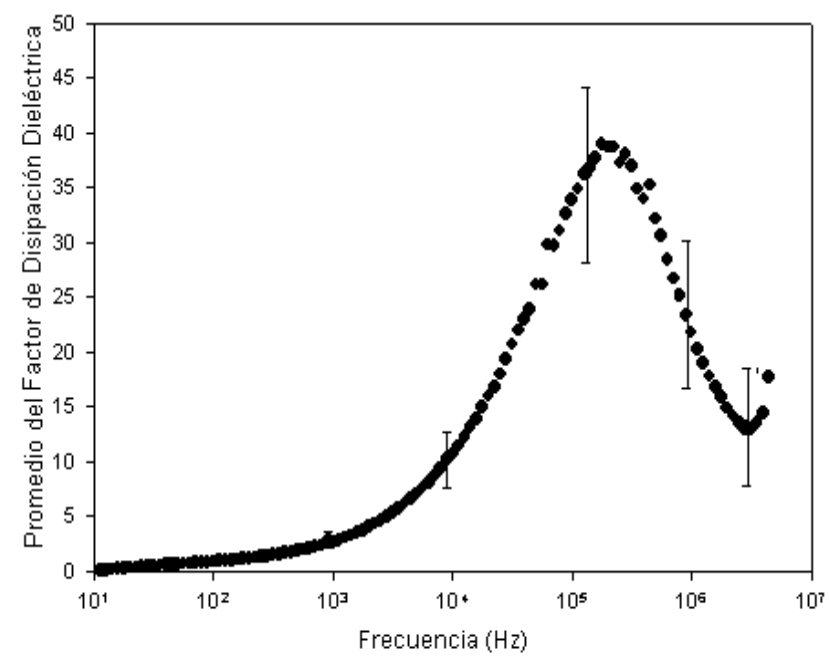

Figura 4.- Promedio del Factor de Pérdida Dieléctrica de las muestras patológicas. 
Factor de disipación dieléctrica.

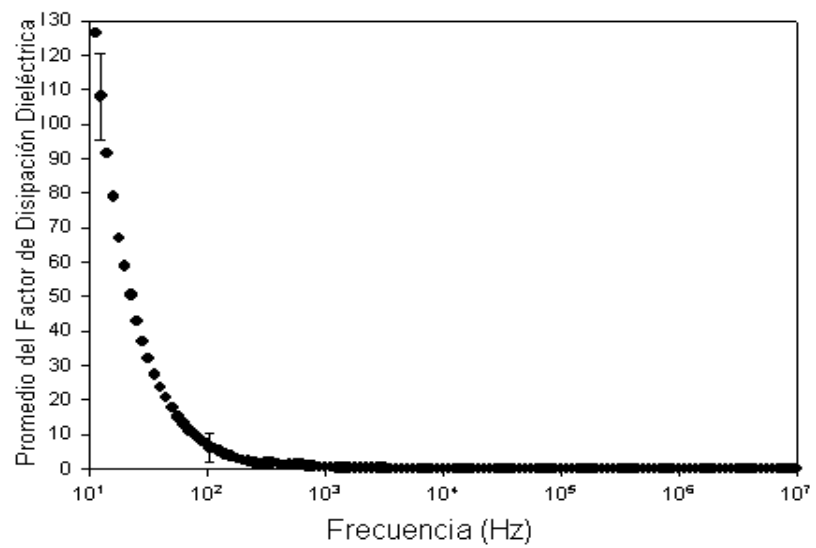

Figura 5.- Promedio del Factor de Pérdida Dieléctrica de las muestras sanas.

estudio las características étnicas, ni antropológicas de los sujetos donadores de las muestras. Esta marcada diferencia entre los resultados obtenidos en cada muestra y visibles en el promedio de las mismas con su factor de dispersión, refleja la dependencia del factor de disipación dieléctrica estudiado sobre la estructura anisotrópica de las muestras, su contenido de agua y electrolitos y su posible relación con el tipo de patología estudiada. Todos estos valores presentan concordancia por los obtenidos por otros autores en estudios relacionados $(5,14-16,18-26)$.

\section{CONCLUSIONES.}

Todas las muestras patológicas reflejan un notable incremento del factor de disipación dieléctrica en un intervalo de frecuencia comprendido entre $10 \mathrm{kHz}$ y $1 \mathrm{MHz}$. Estos resultados nos sugieren la utilización de este dato como una herramienta sensible, quizás para detectar casos patológicos en una temprana edad, capaz de identificar cambios en las propiedades histológicas de tejidos mamarios

Cuadro 2

Valores numéricos del factor de disipación dieléctrica de las muestras con lesión.

\begin{tabular}{|c|c|c|c|c|c|c|c|c|c|}
\hline Muestras & 9 & 10 & 11 & 12 & 13 & 14 & 15 & 16 & Promedio \\
\hline $1,00 \mathrm{E}+02$ & 0,04962395 & 0,05621341 & 0,06572709 & 0,06444669 & 0,05235054 & 0,07168789 & 0,05724147 & 0,05418448 & 0,05893444 \\
\hline $1,00 \mathrm{E}+03$ & 0,67555812 & 0,70956415 & 1,04563533 & 1,01108172 & 0,77881261 & 1,22222336 & 0,84321848 & 0,8577364 & 0,89297877 \\
\hline $1,00 \mathrm{E}+04$ & 2,12489491 & 2,23576166 & 3,79746614 & 3,30029902 & 2,11443486 & 3,3812484 & 2,1548521 & 2,29981467 & 2,67609647 \\
\hline $1,00 E+05$ & 10,1041835 & 10,106452 & 13,3232047 & 15,4537811 & 9,25488986 & 10,7125644 & 7,4846005 & 9,28337925 & 10,7153819 \\
\hline $1,00 \mathrm{E}+06$ & 25,1805728 & 24,2866776 & 31,4836437 & 47,4189834 & 37,6537321 & 36,9747553 & 28,1665362 & 40,1467747 & 33,9139595 \\
\hline $\mathbf{1 , 0 0 E + 0 7}$ & 14,7374529 & 13,6356892 & 23,3056655 & 33,030663 & 18,1884944 & 29,0137166 & 20,2162729 & 22,0011846 & 21,7661424 \\
\hline
\end{tabular}

Cuadro 3

Valores numéricos del factor de disipación dieléctrica de las muestras sin lesión.

\begin{tabular}{ccccccccccc}
\hline Muestras & $\mathbf{9}$ & $\mathbf{1 0}$ & $\mathbf{1 1}$ & $\mathbf{1 2}$ & $\mathbf{1 3}$ & $\mathbf{1 4}$ & $\mathbf{1 5}$ & $\mathbf{1 6}$ & Promedio \\
\hline $\mathbf{1 , 0 0 E}+\mathbf{0 2}$ & 298,924924 & 280,07869 & 30,2215105 & 15,3727118 & 13,2179593 & 2,23279865 & 2,21535491 & 368,634689 & 126,36233 \\
& & & & & & & & & & \\
$\mathbf{1 , 0 0 E}+\mathbf{0 3}$ & 10,3958824 & 9,50975176 & 7,08913572 & 6,57041847 & 5,83442143 & 0,97646879 & 1,91602779 & 13,8653286 & 7,01967937 \\
$\mathbf{1 , 0 0 E}+\mathbf{0 4}$ & 0,20453515 & 1,59083003 & 0,22386744 & 0,23819496 & 0,35226878 & 1,2503927 & 0,1028447 & 0,28430707 & 0,53090511 \\
$\mathbf{1 , 0 0 E}+\mathbf{0 5}$ & 0,12335096 & 0,13840225 & 0,06936552 & 0,08732609 & 0,12335096 & 0,12335096 & 0,00740601 & 0,00079787 & 0,08416883 \\
$\mathbf{1 , 0 0 E}+\mathbf{0 6}$ & 0,10993641 & 0,12335096 & 0,06182204 & 0,07782928 & 0,10993641 & 0,10993641 & 0,13840225 & 0,00740601 & 0,09232747 \\
$\mathbf{1 , 0 0 E}+\mathbf{0 7}$ & 0,09798112 & 0,10993641 & 0,05509885 & 0,06936552 & 0,09798112 & 0,09798112 & 0,12335096 & 0,13840225 & 0,09876217 \\
\hline
\end{tabular}




\section{W Fariñas-Coronado, Z Paz, GJ Orta, E Rodrìguez-Denis.}

y su posible relación con el cáncer de mama, presentando una ventana que nos permite continuar con esta investigación para obtener datos que conlleven a diseñar equipos de diagnóstico de cáncer de mama a futuro.

\section{AGRADECIMIENTOS.}

Los autores agradecen al Consejo Nacional de Investigaciones Científicas y Tecnológicas de la República Bolivariana de Venezuela (CONICIT), a la Fundación para el Desarrollo de la Ciencia y la Tecnología de la región Guayana y a la Universidad Politécnica de Puerto Ordaz ( UNEXPO PUERTO ORDAZ) por el apoyo financiero, científico y tecnológico aportado para el desarrollo de esta investigación.

\section{REFERENCIAS.}

1.- Schwan HP. Electrical properties of tissue and cell suspensions. Adv Biol Med Phys 1957; 5:147-209.

2.- Geselowitz DB. On bioelectric potencials in on inhomogeneous volume conductor, Biophys J 1967; 7:111.

3.- Schwan HP. Four-electrode null techniques for impedance measurement with high resolution. The Review of Scientific Instrument 1968; 39 (4):481-5.

4.- Rush S, Driscoll DA. Current distribution in the brain from surface electrodes. Anesth Analg 1968: 47:717-23.

5.- Gabriel S, Lau RW, Gabriel C. The dielectric properties of biological tissues: 3. Models for the frequency dependence. Physics Med Biol 1995; 41:2271-93.

6.- Gabriel S, Lau RW, Gabriel C. The dielectric properties of biological tissues: 2. Measurements in the frequency range $10 \mathrm{~Hz}$ to $20 \mathrm{GH}$. Physics Med Biol 1995; 41:2251-69.

7.- Steller J, Wtorek J. Complex permittivity of breast tumour tissue, X Internacional Conference on Electrical Bioimpedance 1998. Barcelona, Spain. p. 55-63,

8.- Estrela da Silva J, Marques de Sá JP, Jossinet J. Classification of breast tissue by electrical impedance spectroscopy. Med Biol Engin Comput 2000; 38:26.

9.- Hurt WD. Multiterm Debye Dispersion Relations for Permittivity of Muscle. IEEE Transactions On Biomedical Engineering 1985; 32 (1):60-4.

10.- Murray T, Kagawa W. Electrical impedance computed tomography based on a finite element model. IEEE Transaction On Biomedical Engineering 1985; 32 (3):177-84.

11.- Cook R, Saulnier G, Gisser D, Goble J. ACT3: A high-speed, high-precision electrical impedance tomograph, IEEE Transaction on Biomedical Engineering 1994; 41(8):713-21.

12.- Scholz B, Anderson R. On electrical impedance scanning - Principles and simulations. Siemens Electromemedica 2000; 68:35-44.

13.- Blad B. Clinical applications of characteristic frequency measurements: Preliminary in vivo study. Med Biol Eng Comp 1996; 34:362-5.

14.- Smith SR, Foster KR, Wolf GL. Dielectric Properties of VX-2 carcinoma Versus Normal Liver Tissu, IEEE Transactions On Biomedical Engineering 1986; 33 (5):5224.

15.- Pethig R. Dielectric properties of biological materials: Biophysical and medical applications, IEEE Trans Elect Ins 1984; 19 (5):453-75.

16.- Chauveau N, Hamzaoui L. Ex vivo discrimination between normal, fibrocystic and cancerous tissues in human breast surgical biopsies with bioimpedance spectroscopy. X Internacional Conference on Electrical Bioimpedance, Barcelona, Spain; 1998. p. 52-4.

17.- Surowiec A, Stushly S, Barr J, Swarup A. Dielectric properties of breast carcinoma and the sorrounding tissues. IEEE Trans Biomed Eng 1988; 25:257-62.

18.- De Mercato G, García-Sanchez F. Dielectric properties of fluid-saturated bone: A comparison between diaphysis and epiphysis. Med Biol Eng Comput 1988; 26:313-6.

19.- De Mercato G, García-Sanchez F. Correlation between low-frecuency electric conductivity and permittivity in the diaphysis of bovine femoral bone, IEEE Transactions on Biomedical Engineering 1993; 39 (5):523-6.

20.- Böttcher CJF. Theory of electric polarisation. Chicago: Elsevier Publishing Company 1952; p. 345-78.

21.- Fariñas W, De Mercato G, García-Sánchez F. Propiedades eléctricas de la duramadre humana. Universidad Ciencia y Tecnología 1997; No. 1, p. 4-9.

22.- Fariñas W, De Mercato G, García-Sánchez F. 
Simulación de las propiedades eléctricas de la duramadre humana. Avances Recientes en Bioingeniería 1996; p. 5962.

23.- Fariñas W, De Mercato G, García-Sánchez F. Dependencia frecuencial de las propiedades eléctricas de la duramadre humana" Proc. VII Simposio Latinoamericano de Ingeniería Biomédica 1996. p.23-28.

24.- Malich A, Fritsch T, Anderson R, Boehm T, Facius $\mathrm{M}$, Fleck M, et al. Electrical impedance scanning for classifying suspicious bresast lesions: First results. Eur Radiol 2000; 10:1555-61.

25.- Malich A, Boehm T, Facius M, Freesmeyer MG, Fleck $\mathrm{M}$, Anderson R, et al. Differentiation of mammographically suspicious lesions of breast ultrasound, MRI mammography and electrical impedance scanning as adjunctive technologies in breast cancer detection. Clin Radiol 2001; 56:278-83.

26.- Malich A, Fritsch T, Mauch C, Boehm T, Freesmeyer MG, Fleck M, et al. Electrical impedance scanning: A new technique in the diagnosis of lymph nodes in which malignancy is suspected on ultrasound. Br J Radiol 2001; 74:42-7. 